\title{
An IEC 61850 Sampled Values-based Analyzer for Power Quality applications on Smart Substations
}

\author{
Miguel A. Oliván, Alfonso Mareca, Jorge Bruna, and David Cervero
}

\begin{abstract}
This paper introduces a system capable of characterizing on-site digital instrument transformers from the analysis of the IEC 61850/IEC 61869-9 frames injected by the Stand Alone Merging Units (SAMU) located in digital substations. Besides, exploiting the interesting feature of being able to calculate the Power Quality (PQ) from the information sent by the SAMU, the second objective of this work is to implement a suitable computation architecture to monitor distributed PQ nodes. In a first step, this approach relies on the implementation of a IEC 61000-4-30 class-A PQ library on an industrial-based computer. Later, the library is migrated to a more limited platform to identify the maximum number of parallel nodes that the analyzer can process. Several experimental tests were carried out to verify the suitability of the proposed solution. The excellent results of the calibration by comparison between the digital instrument transformer and the analogue reference transformer and the number of parallel nodes that the system can analyze indicate that the proposed low-cost analyzer meets the stringent class-A requirements of the IEC 61000-4-30 for real-time evaluations.
\end{abstract}

Index Terms-Power quality, substation automation, smart grids, measurement techniques, instrument transformers.

\section{INTRODUCTION}

Nowadays, it is clear that power systems are getting more and more complex as the penetration of new technologies of generation, such as those based on renewables energies, makes it less predictable and difficult to control. In this regard, a deep control and observability of the grid is needed as this new situation is affecting the Quality of Supply (QoS) on existent electrical networks [1], [2]. Besides, the rapid growth of nonlinear loads (e.g. power supplies, variable frequency drives, etc.) is also leading to serious problems of PQ. Therefore, it is urgent for power utilities to improve the capabilities of PQ monitoring on relevant nodes of the grid, like primary substation where a great amount of power is exchanged between transport and distribution lines [3]. It is important to note that the first generation of analogue measurement and control systems in power grid substations are approaching the end of their useful lifespan and their replacement is based on digital substation automation solutions as addressed in the IEC 61850 series. Taking advantage of this situation, novel strategies for PQ monitoring can be deployed relying on this standard, and more concretely based on the IEC 61869-9 [4], which provides the rules for the application of the IEC 61850 on digital instrument transformers, and more concretely towards

This project 17IND06 FutureGrid II has received funding from the EMPIR programme co-financed by the Participating States and from the European Union's Horizon 2020 research and innovation programme.

M.A. Oliván, A. Mareca, J. Bruna (e-mail: jbruna@fcirce.es) and D. Cervero are with Fundación CIRCE, Spain. the on-site calibration of digital power transformers. The basic standard followed in this work, in addition to IEC 61850 series, is the IEC 61000-4-30.

Recent works addressed this issue but just from the point of view of the communication protocol. For example, some authors studied the IEC 61850 to propose a new method to detect the consistency of Power Quality Data Interchange Format (PQDIF) and IEC 61850 communications [5] as well as the update of the IEC 61850 Common Information Model (CIM) [6]. Others reported the need of enhancing the logical node support to enable advanced power quality applications [7], but there is a lack of research exploiting the IEC 61850 PQ capabilities towards their implementation on real PQ monitoring platforms. The use of laboratory testing platforms in the last years is also limited, with some approximations in the field of real-time grid perturbation detection [8] but again incomplete from the point of view of PQ observability.

To complete the current scenario, this paper presents the development of a low-cost and portable Intelligent Electronic Device (IED) capable of analyzing the different Sampled Value (SV) streams coming from the deployed digital instrument transformers via SAMU. Thanks to the development and the seamless integration of different IEC 61000-4-30 Ed.3 class-A PQ libraries, the analyzer is also capable of making a realtime calibration of one Equipment Under Test (EUT) against one Voltage Transformer (VT) standard as well as a highperformance assessment of the existing PQ on each SAMU node tied to the substation monitoring layer.

The paper is structured as follows: Section II presents a general explanation of how the Power Quality can be assess by exploting SV in digital substations environments whereas Section III describes in detail the equipment developed. Then, Section IV shows the validation process carried out in a laboratory environment as well as its performance on a real use case. Finally, the conclusions are presented in Section V.

\section{GENERAL APPROACH}

As indicated in the previous section, the aim of this work includes two main aspects: the first one comprises the development of a device capable of making a real-time calibration of large digital instrument transformers installed at the substation busbars by comparison to a traceable VT. This process is illustrated in Figure 1 where one analogue VT, connected to one SAMU, sends digital information through SV to the proposed system. Moreover, the traceable VT is placed near the equipment to be calibrated (typically measuring the same line and close to the EUT to avoid effect line distortions). 


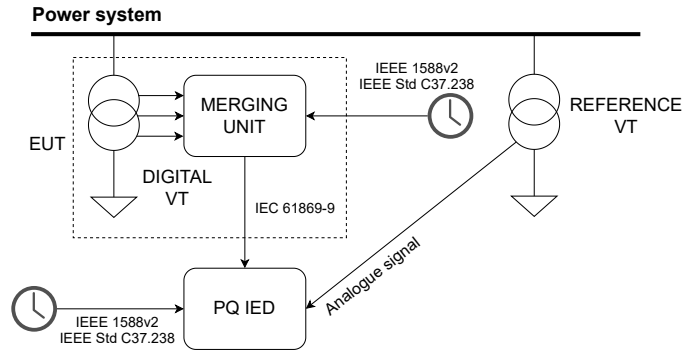

Fig. 1. Calibration of digital instrument transformers by comparison.

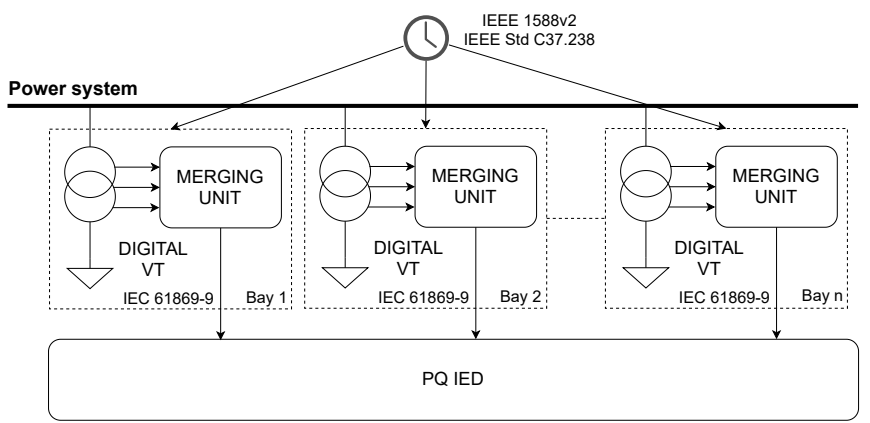

Fig. 2. Multi-point IEC 61850 Power Quality assessment.

This traceable voltage transformer standard is connected to the PQ IED, concretely to a 24-bit high-resolution input with a sampling rate of $50 \mathrm{kSPS}$. Both systems (the EUT and the reference VT) are synchronized by the same IEEE $1588 \mathrm{v} 2$ time source to guarantee that the timestamps of both digital and analogue data are comparable and so the derived PQ results.

The second objective is devoted to develop and test a system capable of analyzing tens of different SV streams to obtain the distributed PQ within the smart substation. The core of this analysis relies on the design of different IEC 61000-4-30 class-A libraries to be implemented in the IED. Concretely, the algorithms implemented followed the rules included in [9], including the following: power frequency, magnitude of the supply voltage, flicker [10], voltage dips, swells and interruptions, voltage harmonics/interharmonics [11], RVCs and voltage unbalance. Special attention was paid to the aggregation process as each SV stream must be analyzed individually as an isolated three-phase system to ensure a proper comparison between SAMUs. In this regard, every SAMU must be synchronized by a common time source. An illustrative general block diagram of an installation with distributed SAMU and their connection to the proposed system is shown in Fig. 2.

As stated before, the IEC 61850 can be used to enhance the interaction with Distributed Energy Resources in an electrical substation. This approach can be used in the frame of Power Quality analysis through the use of IEC 61850 Sampled Values that allows to share the waveform taken by an instrument to the local network. The IEC 61869-9 [4] restricts the Sampled Values flexibility by providing an standardized way to transmit three-phase waveforms, including sampling rates, Application Service Data Unit (ASDU) formats and number of ASDUs per frame. In particular, a sampling rate of $14400 \mathrm{~Hz}$ and 6 ASDU per frame is the preferred configuration for three-phase voltage and current Power Quality assessments. In this regard, this configuration has been used as reference for the work presented in this paper.

On the other hand, precise time synchronization between devices is crucial on distributed power systems as a great timing accuracy must be maintained to decouple data communication from the execution. The strategy is very simple: as every clock plays and important role in the operation of the system and they do not run exactly at the same speed, the more inaccurate clock (slave) is set to the most accurate ones (master clocks or grandmasters), repeating this operation across the whole timing structure recursively. In this regard, with the use of the IEEE1588v02 Precision Time Protocol (PTP) described in [12], [13] it is possible to synchronize distributed clocks with an accuracy of less than $1 \mu s$ via communication networks (e.g. fiber-based ethernet networks) comprising transparent clocks (TC) and boundary clocks (BC). One of the advantages, apart from the accuracy obtained in the synchronization, is that the computational effort demanded on local clocks are low and the bandwidth occupied by this service is almost negligible. This makes this time network protocol a good candidate to be implemented on low-cost and low-energy devices, something important when dealing with distributed measurements in medium to large size power systems.

Regarding the standard that guides the timing requirements on smart substations [13], it specifies a profile named "Standard profile for power system applications" that extend the capabilities of the IEC/IEEE 61850-9-3 for the use of the IEEE 1588 protocol in power installations. Concretely, this profile defines a well-defined subset of IEEE 1588 mechanisms and settings to ensure true interoperability between devices, robust mechanism against network failures and deterministic control of the time delivered across the timing network. These functions are met by the use of two type, length and values (TLVs). More information about the implementation of this profile can be found on [13]. Derived from all these previous considerations, Table I resumes the parameters included in the IEEE C37.238 profile:

TABLE I

IEEE C37.238:2011 POWER PROFILE KEY PARAMETERS.

\begin{tabular}{cc}
\hline Parameter & Value \\
\hline Delay mechanism & P2P \\
VLAN priority & Mandatory $($ default $=4)$ \\
Ethertype & 0x88f7 \\
Announce period & $1 \mathrm{~s}$ \\
Sync period & $1 \mathrm{~s}$ \\
Pdelay period & $1 \mathrm{~s}$ \\
PTP mode & Transparent \\
\hline
\end{tabular}

Besides, the IEC 61850-9-2:2011 [14] defines an attribute (SmpSynch) in SV messages to provide information about time quality and integrity (see Table II). Concretely, the standard indicates that if a merging unit is synchronized to a highaccuracy global area clock, the attribute shall be 2 . If the merging unit is synchronized to a high-accuracy local area clock, this attribute will be 1 whereas the merging unit receives 
the unique identifier of the specific local area clock, the SmpSynch attribute will range from 5 to 254. This framework is fully compatible with all existing Merging Unit (MU)s in the market as these systems receive and process Precision Time Protocol (PTP) messages via IEC 61850 process bus networks according to [14]. Again, this configuration was the preferred one.

TABLE II

SMPSYNCH ATTRIBUTE VALUES.

\begin{tabular}{cc}
\hline Time source & SmpSynch value \\
\hline Global Area Clock & 2 \\
Local Area Clock with unique identifier & $5-254$ \\
Local Area Clock & 1 \\
Absence of Time synchronization & 0 \\
\hline
\end{tabular}

\section{IED ARCHITECTURE AND EXPERIMENTAL SETUP}

A Power Quality platform-independent C99 library (PQ Library) was designed and implemented without any external dependencies. It is able to run on processors and microprocessors of different architectures (Linux, Windows, FreeRTOS, etc.) from desktop computers to low-cost single-board microcomputers like the Raspberry Pi 4 Model B with PTP software timestamping. Besides, the library is flexible to be run in both sequential or task-based execution since the main aggregation groups can be called independently. With a simple configuration based on the sampling frequency and PQ thresholds, the Power Quality library generates all class-A aggregation periods from timestamped instantaneous samples as shown in Fig.3.

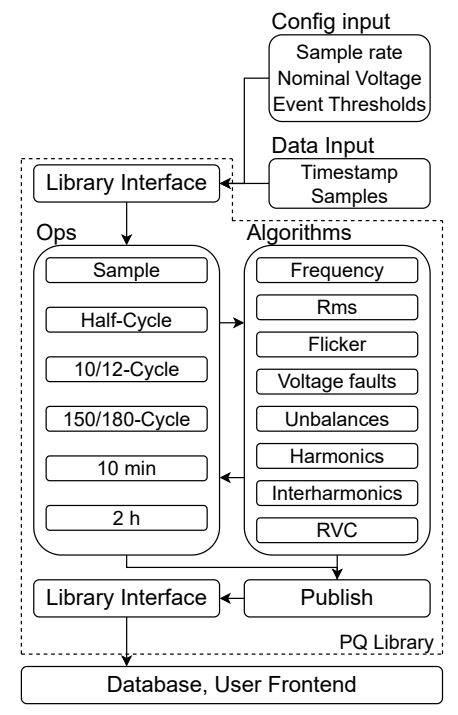

Fig. 3. PQ library architecture.

The IEC 61850 Sampled Values interface of the Real Time Digital Simulator (RTDS) was initially configured following the IEC 61869-9 as described in II. An extra parameter was added to the ASDU, refrTm (refresh time, an absolute timestamp for the sample) to avoid timestamping in the receiving computer given the fact that IEC 61869-9 provides relative timestamping (to the current second) with the smpCnt (sample count) parameter.

To achieve the sub-microsecond time accuracy between the IEEE 1588 grandmaster clock and the presented system, a dedicated Meinberg PTP270PEX card was installed inside the analyzer. This acts as a slave device in the IEEE 1588 network, allowing to set its own PTP seconds and nanoseconds accordingly. The PTP offset calculated by the PTP driver is used to adjust the master oscillator of the PTP270PEX card. In this case, the Meinberg LANTIME M1000 grandmaster houses one dedicated IMS-HPS100 module that provides an accurate IEEE 1588 timing infrastructure for both the SAMUs, through one RTDS, and the proposed PQ system (see Fig. 4).

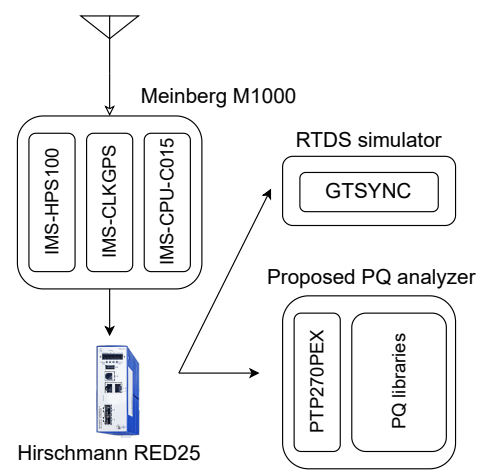

Fig. 4. IEEE1588 timing infrastructure.

In addition to the aforementioned Meinberg systems, the PTP network consisted of a PTP capable Hirchman RED25 switch and a GTSYNC card to provide IEEE 1588 PTP support to the RTDS system. The RTDS GTSYNC card has a fixed configuration of IEEE 1588v2: Network Protocol IEEE 802.3 (Ethernet layer 2), Peer delay as delay mechanism and $250 \mathrm{~ms}^{1}$ of sync interval. The rest of devices in the PTP network, the grandmaster and the PTP270PEX card, were configured accordingly.

Once the timing structure was defined and implemented, it was chosen one traceable and calibrated Fluke 6100A as a reference standard to calibrate both the virtual SAMUs (RTDS) and the PQ analyzer according to the IEC 610004-30 standard. Concretely, the generator was connected to the RTDS simulator's GTIO card and GTNETx2 network interface card. This provides a real-time communication link between to and from the simulator via ethernet. A special firmware option allows converting this GTNETx 2 card as a source of IEC61869-9 sampled value messaging for voltage and currents, which is basically the behaviour of a SAMU. Connection and configuration details for the subsystems used in this setup can be shown in Fig.5, Fig.6 and Fig.7:

\section{LABORATORY RESULTS}

This section presents the results obtained in the laboratory. Test data and results were stored in a PostgreSQL database to ease the evaluation of the IED performance. In addition,

\footnotetext{
${ }^{1}$ Time configuration exceeding the Sync period value recommended by the IEEE C37.238:2011 Power profile (see Table I)
} 


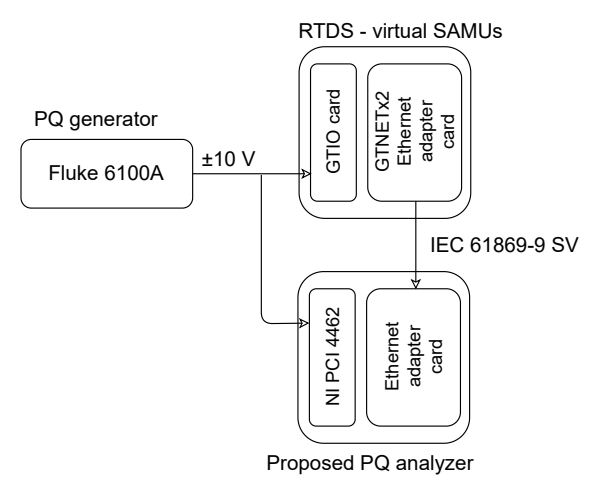

Fig. 5. Calibration of (virtual) SAMUs by comparison with an analogue signal (reference VT).

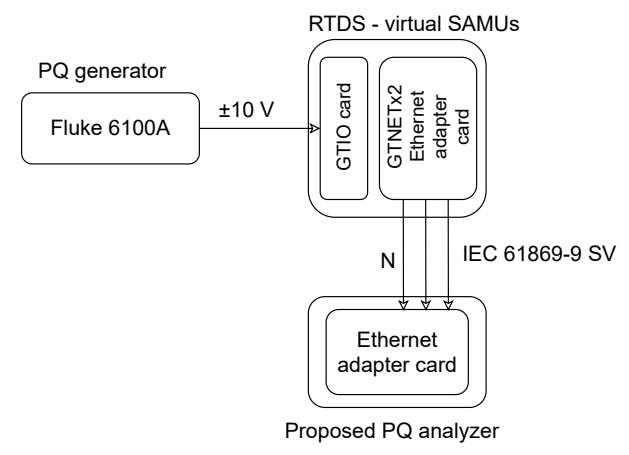

Fig. 6. Paralell PQ analysis of N (virtual) SAMUs .

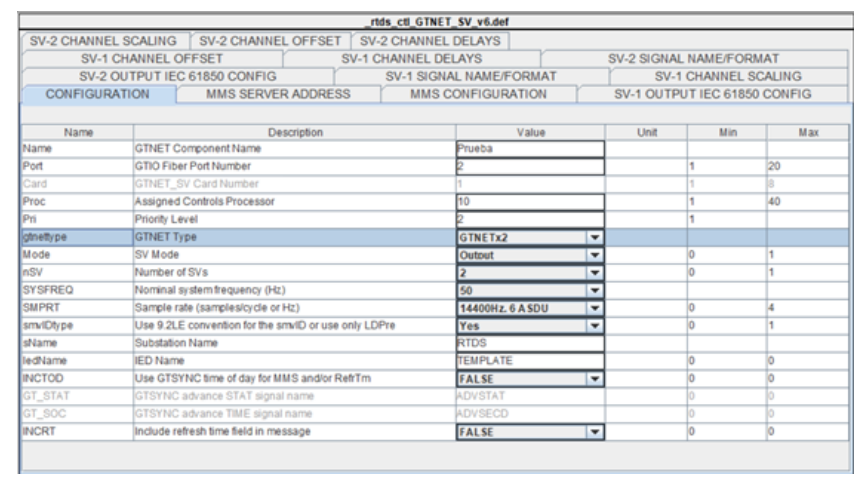

Fig. 7. GTNETx 2 card configuration for IEC 61869-9 SV messaging.

a graphical interface (Fig.8) was developed for benchmarking purposes, especially for the first objective of the work (calibration of digital intrument transformers by comparison, Fig.1) although the evaluation of multiple SV frames was also addressed.

In order to validate the schema of comparison of digital and analogue signals (Fig.8), the Fluke 6100A was configure to output a pure sine voltage waveform of $7.071 \mathrm{Vrms}$ and $50 \mathrm{~Hz}$. This signal was later connected to both the RTDS and the PQ analyzer. In this case, only two PQ parameters were benchmarked, power frequency and magnitude of the supply voltage, obtaining the results shown in Table III. The compliance with the IEC 61000-4-30 was verified according to the limits described in the standard, concretely $\pm 10 \mathrm{mHz}$ for frequency and $\pm 0.1 \%$ for voltage $( \pm 0.00707 \mathrm{~V})$.

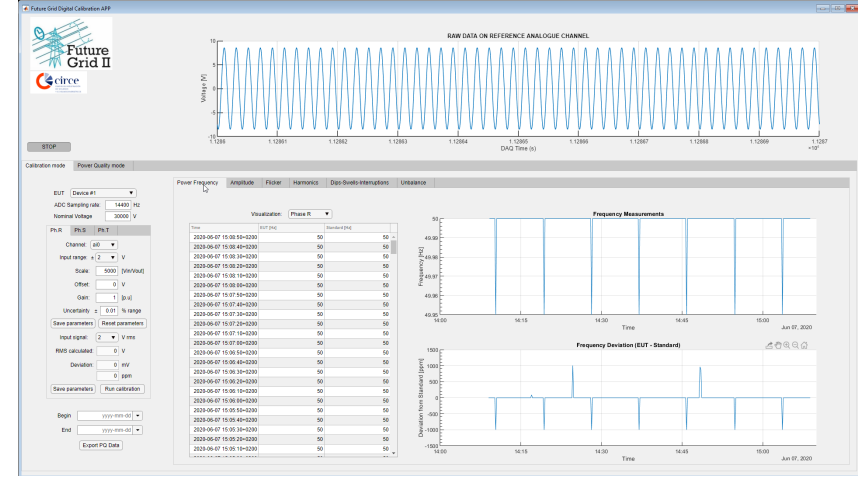

Fig. 8. Interface for the evaluation of results.

TABLE III

RTDS AND PQ ANALYZER BENCHMARKING THROUGH FLUKE 6100A.

\begin{tabular}{cccc}
\hline Fluke 6100A & SV frames & Analogue signal & IEC61000-4-30? \\
\hline $50.000 \mathrm{~Hz}$ & $50.003 \mathrm{~Hz}$ & $50.001 \mathrm{~Hz}$ & Yes \\
$7.0700 \mathrm{~V}$ & $7.0732 \mathrm{~V}$ & $7.0721 \mathrm{~V}$ & Yes \\
\hline
\end{tabular}

Regarding compatibility between the two procedures of measurement, the compatibility index $C I$ was calculated according to Eq.1, where $S V_{\text {meas }}$ is the result obtained from the analysis of the SV stream, Analogue meas $_{\text {is }}$ is the result from the analogue signal and $U_{\text {fluke }}$ the expanded uncertainty of the generator. Considering that the most remarkable contribution in terms of uncertainties will be the expanded uncertainty of the generator $(2.5 \mathrm{mHz}$ for frequency and $1.862 \mathrm{mV}$ for voltage), it was obtained the following results: $C I=0.80$ and $C I=0.59$ for frequency and voltage measurements respectively. It is important to note that that two calibrations are compatible if $C I<1$.

$$
C I=\frac{S V_{\text {meas }}-\text { Analogue }_{\text {meas }}}{U_{\text {Fluke }}}
$$

A similar analysis was conducted over the rest of PQ parameters obtaining again compatible results between measurements (digital and analogue) always within the limits established in the IEC 61000-4-30.

It was also tested the performance of proposed system in terms of scalability. This time the library was installed on a lowcost device, the Raspberry Pi 4. Several variables were analyzed to study the limitations of the platform. Concretely, we identify the CPU temperature (without considering any heat sink), free memory and used memory as the most important ones. A full battery of tests was conducted from the analysis of only one SV stream to the maximum number of SV nodes in parallel, considering the most limiting variable, in this case the CPU temperature. Fig.9 shows the influence on the CPU temperature and memory when increasing the number of nodes. In the light of the results, the maximum number of nodes is around 20 because we reach the temperature where the Raspberry $\mathrm{Pi}$ board begin to throttle the processor ( 80 degrees). 


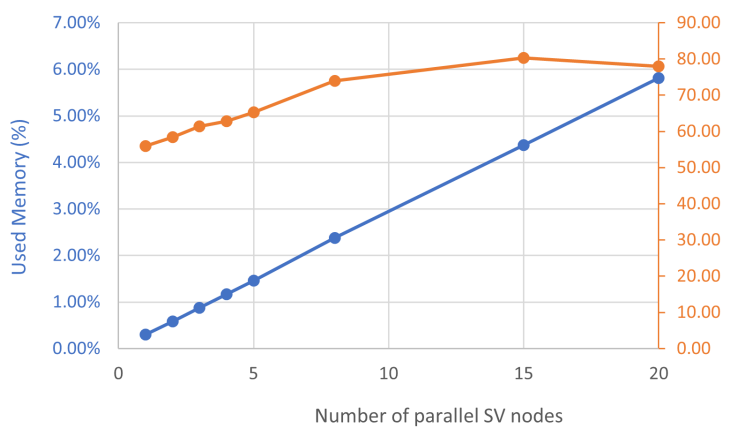

Fig. 9. Influence of parallel nodes on CPU temperature and available memory.

\section{CONCLUSIONS}

The paper presented a novel system based on a sampled values-based architecture to monitor power quality. The analyzer relies on the analysis of different streams of data (analogue and digital) to first calibrate on-site instrument transformers via SAMUs and later to analyze multiple IEC 61850 SV streams from $N$ virtual nodes.

In view of the results, the proposed PQ analyzer meets all the requirements established by the different standards in terms of accuracy for PQ analysis. It was justified that the system can be used for the calibration of digital transformers by comparison with an analogue reference transformer as the PQ results obtained from digital and analogue channels are compatible. On the other hand, the analyzer can analyze up to 20 three-phase nodes in parallel when the PQ library is deployed on a low-cost platform (Raspberry Pi 4). Further nodes could be analyzed in an industrial-based computer with higher resources, as the limiting constraint of the $\mathrm{CPU}$ temperature will not be so crucial due to the existence of active heat sinks.

\section{REFERENCES}

[1] M. Ourahou, W. Ayrir, B. EL Hassouni, and A. Haddi, "Review on smart grid control and reliability in presence of renewable energies: Challenges and prospects," Mathematics and Computers in Simulation, vol. 167, pp. 19-31, 2020. [Online]. Available: https://doi.org/10.1016/j.matcom.2018.11.009

[2] J. P. Braun and C. Mester, "Metrolgy for smart grids," 2018 1st International Colloquium on Smart Grid Metrology, SmaGriMet 2018 , pp. 1-4, 2018.

[3] F. A. F. Suarez, P. Milano, and E. Ragaini, "IEC61850-based Protection System for MV / LV Substations," IEEE Workshop on Power Electronics and Power Quality Applications (PEPQA), pp. 0-4, 2017.

[4] IEC 61869-9:2016 -Instrument transformers - Part 9: Digital interface for instrument transformers, 2016.

[5] W. Jinhao, L. Da, M. Xiaoyang, and Y. Honggeng, "Research on the test of communication protocol of power quality monitoring platform," 2019 IEEE PES Innovative Smart Grid Technologies Asia, ISGT 2019, pp. 3351-3356, 2019

[6] F. Yue, L. Shao, and Z. Wu, "Modeling and implementation of power quality monitoring device based on IEC 61850," China International Conference on Electricity Distribution, CICED, pp. 5-6, 2012.

[7] D. A. Cumming, "Power quality enhancements for IEC 61850," ICHQP 2010 - 14th International Conference on Harmonics and Quality of Power, 2010.

[8] I. M. Moreno-Garcia, A. Moreno-Munoz, V. Pallares-Lopez, M. J Gonzalez-Redondo, E. J. Palacios-Garcia, and C. D. Moreno-Moreno, "Development and application of a smart grid test bench," Journal of Cleaner Production, vol. 162, pp. 45-60, 2017. [Online]. Available: http://dx.doi.org/10.1016/j.jclepro.2017.06.001
[9] IEC 61000-4-30:2015 - Electromagnetic compatibility (EMC) - Part 4-30: Testing and measurement techniques - Power quality measurement methods, 2015.

[10] IEC 61000-4-15:2010 - Electromagnetic compatibility (EMC) - Part 4-15: Testing and measurement techniques - Flickermeter - Functional and design specifications, 2010.

[11] IEC 61000-4-7:2002 - Electromagnetic compatibility (EMC) - Part 4-7: Testing and measurement techniques - General guide on harmonics and interharmonics measurements and instrumentation, for power supply systems and equipment connected thereto.

[12] IEEE Std 1588:2008 - Standard for a Precision Clock Synchronization Protocol for Networked Measurement and Control Systems, 2008, vol. 2008, no. July.

[13] C37.238:2017 - IEEE Standard Profile for Use of IEEE 1588 Precision Time Protocol in Power System Applications, 2011, vol. 2017, no. July.

[14] IEC 61850-9-2:2011 - Communication networks and systems for powe utility automation - Part 9-2: Specific communication service mapping (SCSM) - Sampled values over ISO/IEC 8802-3, 2011.

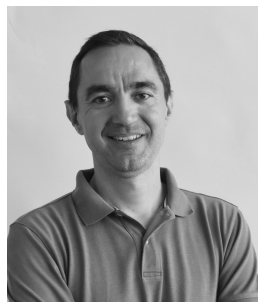

Miguel Á. Oliván Ph.D. in Physics (2016), MsC in Systems and Computer Science (2012) from the University of Zaragoza. Twenty years of experience in software development focused mainly in data acquisition systems, data analysis and Supervisory Control And Data Acquisition (SCADA) systems. His main role in CIRCE is the design and development of Smart Grids SCADA systems and their integration with remote managing algorithms. Additionally, his role includes the design and implementation of PQ software on smart PQ analyzers.

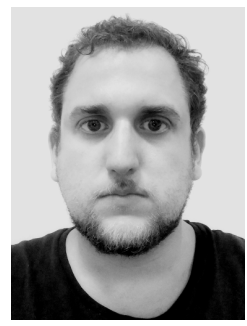

Alfonso Mareca received his B.Sc. degree in mechatronic engineering from the University of Zaragoza in 2019. He is a researcher and developer in the field of PQ for mcu's for the Research Centre for Energy Resources and Consumption (CIRCE). He is currently studying a M.Sc. degree in electronic engineering His fields of interest are data acquisition electronics as well as electrical mobility. He has competed in the engineering Motostudent Electric IV and V contest. He won the Tecnalia technological innovation award with his final B.Sc. project in 2019 .

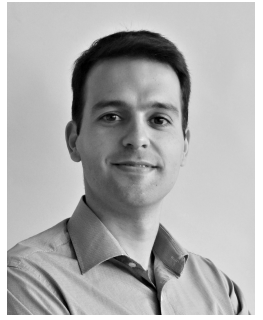

Jorge Bruna received his M.Sc. and Ph.D. degree in electrical engineering from the University of Zaragoza, Spain, in 2011 and 2016, respectively. He has been a Researcher with the LME-Research Centre for Energy Resources and Consumption (CIRCE), University of Zaragoza, since 2008. He is leading the accredited LME-CIRCE laboratory since 2019. $\mathrm{He}$ has authored several scientific papers in the field of PQ and developed advanced measurements algorithms for monitoring power quality parameters

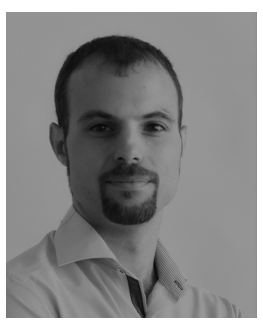

David Cervero received his bachelor's degree in Industrial Engineering (specialisation in electric systems), and his master's degree in Renewable Energies and Energy Efficiency from the University of Zaragoza in 2009 and 2011, respectively. He is the Head of Electrical Grids Supervision Systems in CIRCE Foundation. He has a deep expertise in $\mathrm{PQ}$, data acquisition and digital signal processing, developing dedicated software for measuring PQ in electrical distribution networks. Fault location and monitoring in electrical distribution networks, with application in predictive maintenance, are also his current research fields. 